Patricia Pavón-León ${ }^{1}$ Armando J. Martínez ${ }^{2}$ Gaudencio Gutiérrez-

Alba $^{3}$

Sealtiel Armenta-Arellano ${ }^{4}$

${ }^{1}$ Instituto de Ciencias de la Salud. Universidad Veracruzana. Xalapa, Veracruz México. Correo: ppavon@ uv.mx https://orcid.org/0000-0002$1868-4530$

Instituto de Neuroetología. Universidad Veracruzana. Xalapa, Veracruz México. Correo: armartinez@ uv.mx https://orcid.org/0000-00021248-2516

${ }^{3}$ Instituto de Ciencias de la Salud. Universidad Veracruzana. Xalapa, Veracruz México. Correo: gagutierrez@ uv.mx https://orcid.org/0000-00032030-758X

${ }^{4}$ Instituto de Ciencias de la Salud.

Universidad Veracruzana. Xalapa, Veracruz México. Correo:sarmenta@ uv.mx https://orcid.org/0000-00019854-4388

Autor de correspondencia: P. Pavón-León

Universidad Veracruzana .Instituto de Ciencias de la Salud Av. Luis Castelazo Ayala s/n Colonia Industrial Animas

Xalapa, Veracruz, México

E-mail: ppavon@uv.mx

DOI: https://doi.

org/10.31052/1853.1180.

v24n1

@Universidad Nacional de Córdoba

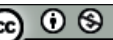

Trabajo recibido: 25 de setiembre 2019.

Aprobado: 3 de febrero

\section{ADULTOS MAYORES POBLACIÓN VULNERABLE EN MÉXICO: UNA APROXIMACIÓN MULTIVARIADA.}

\author{
ELDERLY VULNERABLE POPULATION IN \\ MEXICO: A MULTIVARIATE APP ROACH. \\ IDOSOS POPULAÇÃO VULNERÁVEL NO \\ MÉXICO: UMA ABORDAGEM MULTIVARI- \\ $A D A$.
}

\section{Resumen}

Objetivo: Determinar la contribución de las variables socioeconómicas, estilos de vida, carga de enfermedad y gasto de bolsillo en salud para integrar un perfil multivariado,a fin de caracterizar alos adultos mayores que asisten a instituciones públicas de salud en México, como apoyo al diseño de políticas públicas.Material y métodos. Estudiotransversal analítico. Población de estudio, adultos mayores usuarios de la consulta externa de tres unidades públicas de salud. Se utilizó técnica multivariada de componentes principales. Resultados. Se tipificaron cuatro grupos: aquéllos con estilos de vida malos,gasto de bolsilloalto, padecían múltiples enfermedades, nivel socioeconómico bajo, casadosy/o cohabitaban con otras personas en el hogar, estos grupos fueron hombres jefes de familia y mujeres no jefas de familia. En cambio, quienes presentaron mejores estilos de vida, gasto de bolsillo menor, nivel socioeconómico regular y viven solos, fueron grupos de hombres no jefes de familia y mujeres jefas de familia.

Palabras clave: Envejecimiento; Estilos de vida; Factores socioeconómicos; Servicios de salud. 
Objective. To determine the contribution of socioeconomic variables, lifestyle, burden of disease and out-of-pocket expenditure on health to integrate a multivariate profile,inorder to characterize older adults attending public healt hinstitutions in Mexico, as support forpolicydesignpublic.Material and methods. An analytic cross-sectional study. Population of study, older adults who attend out-patient care in three medical facilities. Multivariatetechnique of maincomponentswasused. Results. Fourgroupsweretypified: thosewithbadlifestyles, highout-of-pocket expenses, sufferedmultipleillnesses, lowsocioeconomic status, married and / orcohabitedwithotherpeople in the home, thesegroupsweremaleheads of household and womennotheads of household .Ontheotherhand, thosewhopresentedbetterlifestyles, lowerout-of-pocket expenses, regular socioeconomic status and livealone, weregroups of menwhowerenotheads of household and womenheads of households.

Key words: Aging; Lifestyle; Socioeconomic factors; Health services.

\section{Resumo}

Objetivo. Determinar a contribuição de variáveis socioeconômicas, estilos de vida, carga de doenças e despesas diretas com saúde para integrar um perfil multivariado, a fim de caracterizar os idosos que freqüentam instituições de saúde pública no México, como apoio ao desenho de políticas públicas de saúde no México. Material e métodos. Estudo transversal analítico. População da pesquisa, idosos usuários da consulta ambulatorial de três unidades de saúde pública. Foi utilizada a técnica multivariada de componentes principais. Resultados. Quatro grupos foram tipificados: aqueles com estilos de vida ruins, gastos diretos elevados, sofriam de doenças múltiplas, baixo nível socioeconômico, casados e / ou convivendo com outras pessoas em casa, esses grupos eram chefes de família masculinos e as mulheres não eram chefes de família. . Em vez disso, aqueles que apresentavam melhores estilos de vida, gastos mais baixos, nível socioeconômico regular e viviam sozinhos eram grupos de homens que não eram chefes de família e mulheres que eram chefes de família.

Palavras chave: Envelhecimento; Estilos de vida; Fatores socioeconômicos; Serviços de saúde.

\section{Introducción}

La salud está determinada, entre otros factores, por las condiciones socioeconómicas que a su vez influyen en los estilos de vida como los hábitos alimenticios, la actividad física, el estrés, el consumo de alcohol y drogas(1)(2)(3)(4)(5)(6). Además, cambios en la salud puedenafectar su posición social al poner en peligro las oportunidades de empleo e ingresos, creando un círculo vicioso entre determinantes e inequidades en salud. Por lo tanto, el estado de salud de un adulto mayor está relacionado con los determinantes sociales y el estilo de vida que desarrolló a lo largo de su vida.

La población de adultos mayores (AM) en México está creciendo de forma acelerada. Ésto debido a quela tasa de natalidad y mortalidad infantil ha disminuido y la esperanza de vida ha aumentado, como consecuencia del incremento de la cobertura en salud y la introducción de intervenciones eficaces en el control y tratamiento de enfermedades infecciosas(7). Como efecto delas transicionesdemográfica y epidemiológica, el sistema de salud mexicano enfrenta el reto del envejecimiento poblacional, lo cual se traduce en una mayor morbilidad y mortalidad por enfermedades crónicas(8).Para atender estas necesidades de salud, las cuales progresivamente demandarán servicios no previstos e implicarán mayores gastos en salud, dicho sistema no está preparado(9). 
En este nuevo panorama, la utilización de servicios públicos de salud en algunas ocasiones genera a las familias erogaciones directas, las cuales se les denomina gasto de bolsillo en salud (GBS), mismas que son efectuadasdirectamente por las personas para pagar la atención al momento de enfermar. Es decir, el dinero que desembolsan directamente los hogares (10).

Para los AM el costo de la atención en salud es un aspecto particularmente relevante, especialmente en los países de ingresos medios y bajos. En México, menos de la mitad de los AM está incorporado al sistema de seguridad social. Para los AM sin seguridad social, atendidos por el Ministerio de Salud (SSa)la cobertura y protección financiera varían significativamente y el acceso a los servicios de salud sigue siendo una restricción, lo cual los coloca en una situación de vulnerabilidad (11). Por ello, los sistemas de salud deben estar atentos a las necesidades de estos grupos vulnerables, haciendo uso de técnicas que relacionen múltiples variables de modo simultáneo, y cuya evidencia ofrezcan las pautas para la toma de decisiones orientada a cumplir con los objetivos de mejorar la salud y garantizar la protección financiera de los mismos.

Considerando lo anterior, el objetivo de este estudio fue determinar la contribución de las variables socioeconómicas, estilos de vida, carga de enfermedad y GBSpara integrar un perfil multivariado, a fin de caracterizar a los adultos mayores que asisten a instituciones públicas de salud en México, como apoyo al diseño de políticas públicas.

\section{Material y Método}

Tipo de estudio: El diseño fuetransversal analítico.La investigación se realizó en tres unidades médicas del sector público: Centro de Salud (CS)de primer nivel de atención;Hospital Regional (HR) de segundo nivel; y Hospital de Alta Especialidad (HAE) de tercer nivel. Todos localizados enuna misma ciudad, pertenecientes a Servicios Estatales de Salud (SESAS) de una Entidad Federativa en México.Participantes: La población de estudiofueronadultos mayores (personas de 60 años y más) usuarios de consulta externaen estas tres unidades médicas. Muestra: Para calcular el tamaño de muestra se utilizó la fórmula para poblaciones finitas con un nivel de confianza de $99 \%$ y un margen de error del $2 \%$. Además, se realizó la estimación de la potencia de la muestra para la asociación de las variables, se obtuvo un valor de 0.79 a 0.81 con un tamaño de muestra de 1217 adultos mayores. La distribución de la muestra se llevó a cabo de forma proporcional en las tres unidades médicas considerando la población atendida el año anterior.Variables: Las que se analizaron fueron: a) Gasto de Bolsillo en Salud (GBS),se estimó como el gasto generado en el mes anterior de los adultos mayores por la atención médica, incluyó honorarios, medicamentos, cirugías, exámenes de laboratorio y gabinete, transfusiones y servicios odontológicos (los valores están estimados en dólares americanos USD); b) Socioeconómica que incluye escolaridad, nivel socioeconómico, tipo de aseguramiento, ingreso y gasto mensual; c) Estilos de vida,definidacomo el conjunto de comportamientos, hábitos o patrones del individuo considerando familia y amigos, actividad física, nutrición, consumo de tabaco, alcohol y otras drogas, horas de sueño, presencia de estrés, tipo de personalidad, pensamientos positivos y satisfacción con las labores que desempeña; d) Sociodemográfica, que refieren a edad, sexo, estado civil, con quién vive, si es jefe de familia (persona que sostiene económicamente al hogar) y ocupación; y e) Enfermedad, padecimientos que presentaban los adultos mayores en el momento de la encuesta.

Instrumentos: Para estimar el GBS, se diseñó un instrumento ex profeso para el estudio (20 items, Alfa de Cronbach: 0.70). Se evaluaron variables socioeconómicas con el Índice de Nivel Socioeconómico (INSE) (12), para población mexicana que integra variables como las condiciones de la vivienda (material del piso, agua potable, eliminación de excretas e índice de hacinamiento) y el número de años estudiados por el jefe de la familia. Además 
del INSE, se incluyó el tipo de aseguramiento, ingreso (salario y/o jubilación, y/o apoyo económico) y gasto mensual (alimentación, renta, luz, agua, teléfono y esparcimiento). Para medir el estilo de vida se utilizó el cuestionario FANTASTIC, integrado por 25 ítems cerrados que exploran nueve dominios físicos, psicológicos y sociales, cada ítem tiene una escala tipo Likert ( 0 a 4 ), donde $0 \leq 39$ equivale a existe peligro, 40 a 59 malo, 60 a 69 regular, 70 a 84 bueno y excelente de 85 a 100 puntos (instrumento validado para población mexicana) (13).Además, las características sociodemográficas se evaluaron con un instrumento diseñado ex profeso para el estudio ( 7 items, Alfa de Cronbach: 0.70).

Procedimiento: Se realizó una encuestacada adulto mayor, en la cual se le proporcionóla información pertinente acerca del objetivo del estudio y los individuos que aceptaron, se les aplicó un cuestionario estructurado. Para estandarizar el registro de la información,se les entregó a los encuestadores un manual sobre el uso del instrumento y fueron capacitadospara su aplicación por el investigador responsable.

Análisis estadístico

El análisis estadístico incluyó estadística descriptiva de las variables en escala porcentual y se usó Chi-cuadrada para comparar proporciones. Debido a la estructura multidimensional del estudio se realizó un análisis de componentes principales (CP), para obtener la ordenación multivariante(14) la cual aportó un conjunto de indicadores de la combinación lineal de las variables que se ordenaron respecto al porcentaje de varianza que explicó cada CP. Los "factor score" de cada individuo que representa el índice multivariantefue analizado con un Modelo Lineal Generalizado (MLG), con un ajuste de ANCOVA con el siguiente diseño: $\mathrm{y}=$ Unidad médica + Grupo + Covariable $(\mathrm{CP} 1)+$ Grupo x Covariable + error, y sus respectivas interacciones, donde " $y$ " corresponde a los factor score (índices multivariantes) obtenidos en el CP2 a CP5; la unidad médica con sus tres niveles; el grupo con cuatro niveles; el CP1 se consideró como la covariable. Se verificó cumplir con la normalidad multivariante y en los supuestos del análisis de ANCOVA, no fue necesario verificar normalidad y homogeneidad de varianzas tal como lo indica Gorsuch(15).

Consideraciones éticas: El protocolo de investigación fue aprobado por los comités de ética y de investigación de las unidades médicas. Número de aprobación: CEM24/13. Previo a la aplicación se obtuvo el consentimiento informado por escrito de cada participante. Además, se aseguró la confidencialidad y el anonimato de la información proporcionada por los pacientes.

\section{Resultados}

Descripción socio demográfica y económica: La muestra se conformó por 1217 adultos mayores. La proporción de mujeres fue de $61 \%$ y de hombres $39 \%$, y la edad promedio fue $78 \pm 8$ años. Asimismo, más del 50\% de los adultos mayores provenían de localidades rurales (menosde2500 habitantes) y $75.6 \%$ no concluyeron los estudios de primaria (menos de 6 años de estudio). La tasa de respuesta del estudio fue de $97.7 \%$.

El 26.3\% tenían empleo, las ocupaciones más frecuentes fueron vendedor ambulante, campesino, servicio doméstico, comerciante, oficinista y albañil. La mayoría de los participantes $(94.3 \%$ ) no contaban con una pensión por jubilación y $63.5 \%$ tuvieron algún apoyo económico extra a su salario o jubilación, como programas federales o estatales o de familiares. El 93\% de los adultos mayores tenían algún tipo de aseguramiento y el $86.2 \%$ estaban afiliados al Seguro Popular.

Las variables: con quién vive, jefe de familia, estado civil, aseguramiento, enfermedad, estilos de vida, nivel socioeconómico, ingreso mensual, gasto mensual y GBS mensual, indicaron variación entre hombres y mujeres. El $67.2 \%$ de los hombres y $65.1 \%$ delas mujeres vivían con algún familiar $(\mathrm{p}=0,001)$. Además, cinco de cada 10 hombres eran jefes de familia $(\mathrm{p}=0,001)$ mientras que pocas mujeres realizan dicha función (Tabla 1$)$. 
Los estilos de vida oscilaron entre bueno a regular, el nivel socioeconómico de medio a alto, el ingreso y el gasto mensual fueron similares aunque el GBS fue más alto en los hombres. Respecto a la carga de enfermedad $63,6 \%$ de los hombres y 75,6\% de las mujeres padecían hipertensión arterial y diabetes $(\mathrm{p}=0,001$, Tabla 1$)$.

Tabla $N^{\circ}$ 1: Caracterización de los adultos mayores comparando hombres y mujeres.

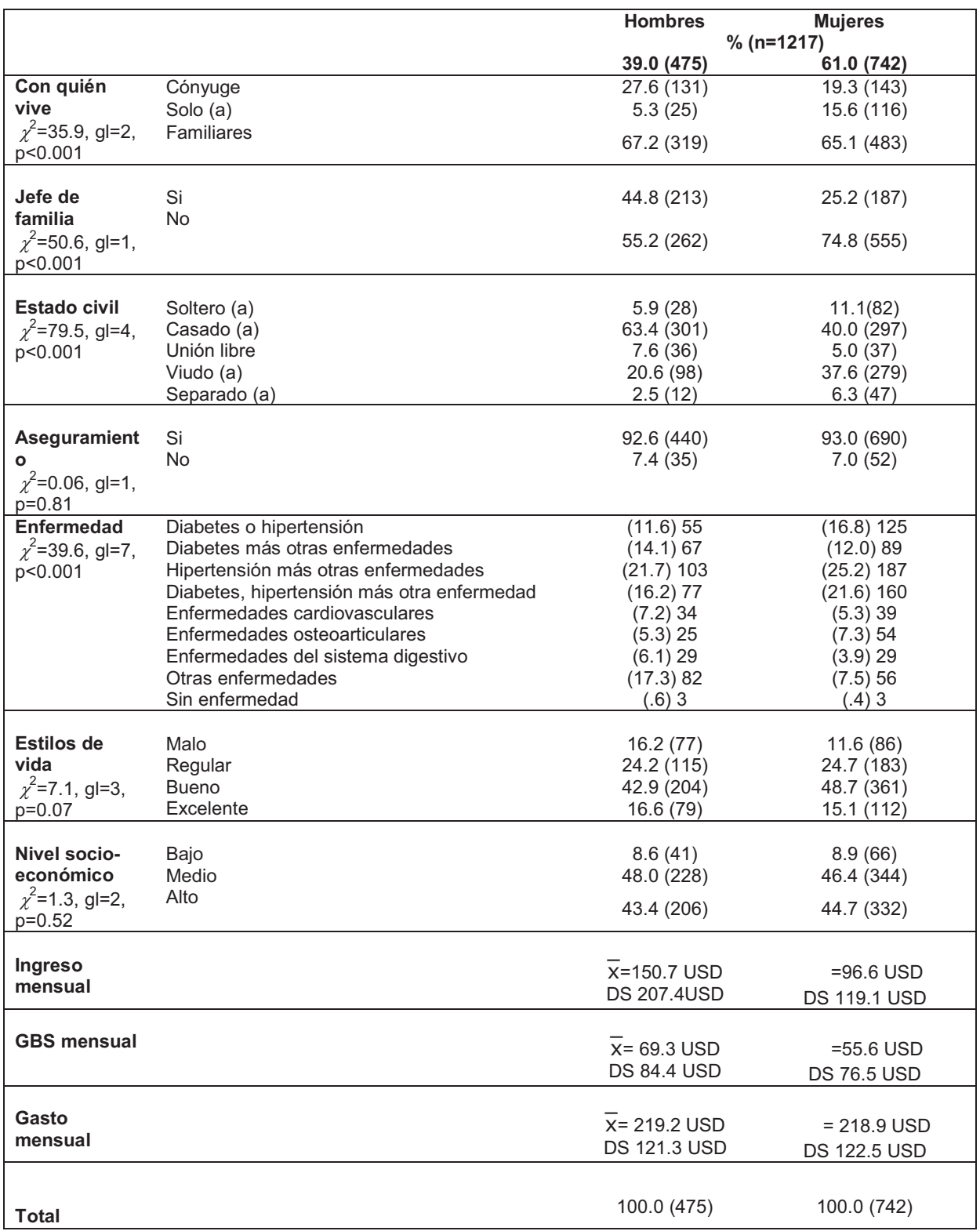

Asociación multivariante: La combinación lineal de las variables para CP1 contribuyó con $19 \%$ de la varianza explicada y se integró por las variables ingreso mensual y jefe de familia; el CP2 aportó el 14\% y la combinación fue con quién vive, nivel socioeconómico y enfermedad;el CP3, también contribuyó con 14\% las variables asociadas fueron GBS y estilos de vida; en cambio el CP4 aportó el 12\% con el GBS y estado civilasociados, y el CP5 contribuyó con 12\% que incluye el nivel socioeconómico y enfermedad (Tabla 2). 
Tabla N²: Autovalores de los componentes principales CP1 a CP5 con la contribución de las variables a partir de la combinación lineal que se explicó con el $71 \%$ de la varianza.

\begin{tabular}{|llrrrrr|}
\hline Variables & & CP 1 & CP 2 & CP3 & CP4 & CP 5 \\
\hline Sociodemográficas & Jefe familia & $\mathbf{0 . 8 0}$ & 0.12 & -0.25 & 0.25 & -0.11 \\
& Estado civil & 0.43 & -0.23 & 0.29 & $\mathbf{- 0 . 5 2}$ & -0.07 \\
& Con quién vive & -0.31 & $-\mathbf{0 . 6 1}$ & 0.08 & -0.30 & -0.005 \\
\cline { 2 - 7 } Socioeconómicas & Gasto de bolsillo en salud & 0.17 & 0.15 & $\mathbf{0 . 5 9}$ & $\mathbf{0 . 5 6}$ & -0.04 \\
& Ingreso mensual & $-\mathbf{0 . 7 7}$ & -0.32 & 0.20 & 0.03 & -0.11 \\
Salud & Nivel socioeconómico & 0.06 & $\mathbf{- 0 . 6 2}$ & -0.06 & 0.38 & $\mathbf{- 0 . 5 5}$ \\
\cline { 2 - 7 } & Estilos de vida & -0.14 & -0.10 & $\mathbf{- 0 . 7 5}$ & 0.09 & -0.03 \\
& Enfermedad & -0.10 & $\mathbf{0 . 5 0}$ & 0.01 & -0.31 & $\mathbf{- 0 . 7 6}$ \\
& Expl.Var & 1.59 & 1.23 & 1.13 & 0.99 & 0.93 \\
& \% Total & $\mathbf{0 . 1 9}$ & $\mathbf{0 . 1 4}$ & $\mathbf{0 . 1 4}$ & $\mathbf{0 . 1 2}$ & $\mathbf{0 . 1 2}$ \\
\hline
\end{tabular}

Los valores en negritas marcadas para cada variable representan la carga factorial considerando un valor superior a 0.50

Es importante destacar que la ordenación multivariada conformó cuatro grupos ordenados por sus características a partir de la dispersión de los índices "factor score" obtenidos en el CP1 y CP2, los cuales corresponde a las variables ser jefes de familia y género. En detalle, el Grupo 1 se integró por mujeres no jefas de familia; Grupo 2= hombres no jefes de familia; Grupo 3= hombres jefes de familia y Grupo 4= mujeres jefas de familia (Figura 1). Por ello en modelos de ANCOVA para modelar los índices de losCP2, CP3, CP4 y CP5, fueron analizados integrando al diseño el Grupo como una nueva variable nominal y la covariable correspondió al CP1 en todos los ajustes.

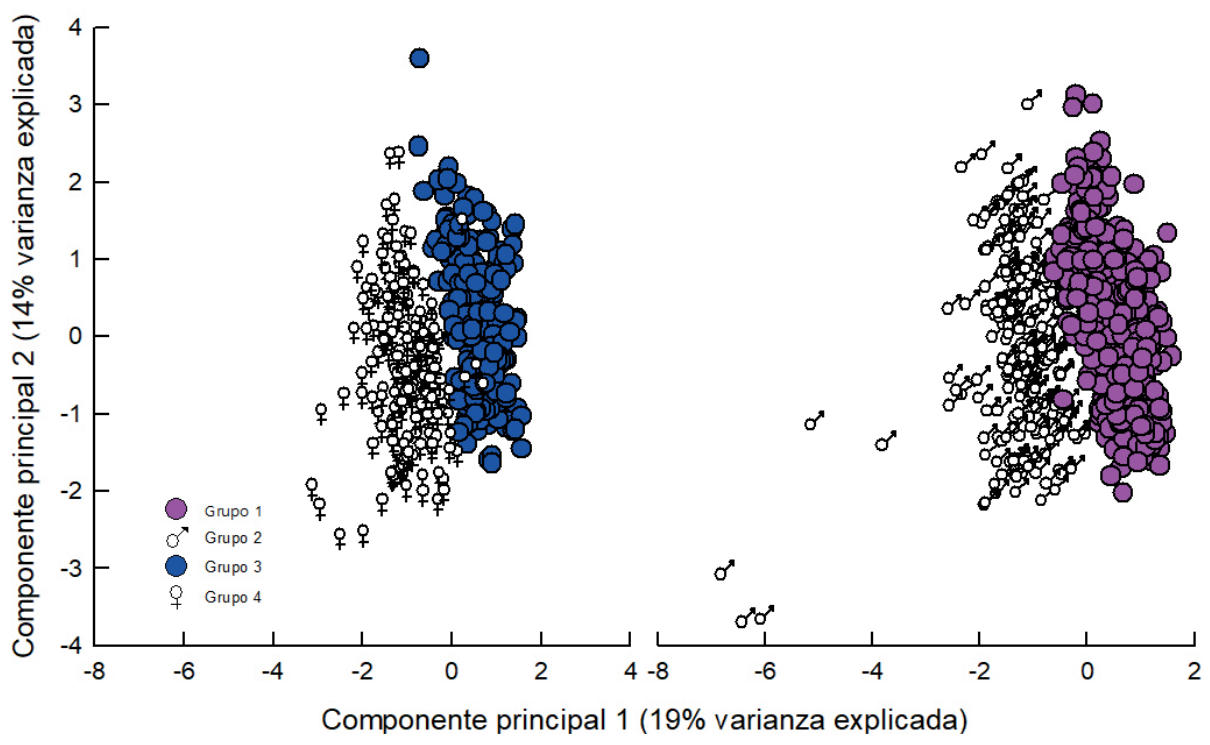

Figura $\mathbf{N}^{\circ} 1$ : Dispersión de los índices de cada adulto mayor que representa a los componentes principales (CP 1 y CP2) de las variables jefe de familia y sexo en los cuatro grupos obtenidos por el Clousteranálisis:Grupo 1: mujeres no jefas de familia, Grupo 2: hombres no jefes de familia, Grupos 3: hombres jefes de familia y Grupos 4: mujeres jefas de familia. 
Comparación entre grupos

ElCP2, que se integró por las variables con quién vive el adulto mayor, nivel socioeconómico y el número de enfermedades, mostró contraste entre los valores promedio al comparar entre las unidades médicas(F2, 119=33,35 $\mathrm{p}<=0,0001$, Tabla $3 \mathrm{~A})$ y diferencias en los cuatro grupos de adultos mayores $(\mathrm{F} 3,119=24,75 \mathrm{p}<=0,0001$, Tabla $3 \mathrm{~A})$. La triple interacción entre unidades médicas $\times$ cuatro grupos $\times$ la covariable CP1 (ANCOVA)indica que el Grupo 1 (mujeres no jefas de familia) y el Grupo 3 (hombres jefes de familia) tuvieron los valores promedio más altos en las tres unidades médicas, en el número de enfermedades y en que los adultos mayores cohabitaran con otros miembros de la familia en el hogar. En cambio, el Grupo 2 (hombres no jefes de familia) y Grupo 4 (mujeres jefas de familia) en launidad médica CS y en HR tuvieron los valores promedio más bajos que representaba soloun tipo de enfermedad, hipertensión o diabetes yademás estos adultos mayores vivían solos(ANCOVA F2,119=27, $\mathrm{r} 2=0.30, \mathrm{p}<=0,0001$ Figura 2A).

Tabla N 3: Fuente de variación del ANCOVA para el análisis de los "factor score" de los componentes principales $\mathrm{CP} 2$ a $\mathrm{CP} 5$ que se usaron como variables de respuesta. Además se indican $\mathrm{F}$ y $\mathrm{P}$ para el modelo con sus factores fijos e interacciones, y el CP1, representa la covariable.

\begin{tabular}{|c|c|c|c|c|}
\hline & CP2 & CP3 & $\mathrm{CP} 4$ & CP5 \\
\hline Efecto & $\mathrm{F}$ & $\mathrm{F}$ & $\mathrm{F}$ & $\mathrm{F}$ \\
\hline Unidad médica & $33.35^{\star *}$ & $10.50^{* *}$ & 0.70 & $4.83^{*}$ \\
\hline Cuatro grupos & $24.75^{\star \star}$ & $21.57^{\star \star}$ & $19.64^{\star \star}$ & $4.93^{*}$ \\
\hline Unidad médica $\times$ Cuatrogrupos & $13.16^{* *}$ & $6.651^{\star *}$ & $3.79^{* *}$ & 1.40 \\
\hline Unidad médica $\times$ Cuatro grupos $\times \mathrm{CP} 1$ & $26.78^{\star *}$ & $17.40^{* *}$ & $8.10^{* *}$ & $3.22^{* *}$ \\
\hline
\end{tabular}

El CP3, se integró por los estilos de vida y el GBS. De igual forma se obtuvo contraste al comparar los valores promedio entre unidades médicas (F F2, 119=10,50 $\mathrm{p}<=0,0001$, Tabla 3B) y entre los cuatro grupos (F3, $119=21,57 \mathrm{p}<=0,0001$, Tabla 3B). Además, la triple interacción unidades médicas $\times$ cuatro grupos $\times$ la covariable $\mathrm{CP} 1$ también indicó que en la unidad médicaHAE, los adultos mayores tenían estilos de vida de regular a malos y un GBS alto. Asimismo en las unidades médicas HR y CS los valores promedio son iguales que en el HAE entre el Grupo 1 (mujeres no jefas de familia) y en el Grupo 3 (hombres jefes de familia) presentando estilos de vida de regular a malos y un GBS alto. Los adultos mayores del Grupo 2 (hombres no jefes de familia) y el Grupo 4 (mujeres jefas de familia) en el HR y CS tuvieron mejores estilos de vida y el promedio de GBS fue más bajo (ANCOVA F2,119=17, $\mathrm{r} 2=0,20, \mathrm{p}<=0,0001$ Figura $2 \mathrm{~B}$ ).

El CP4, incluyó al GBS de los adultos mayores y su estado civil. Este componente principal, no aportó contraste entre unidades médicas (F2, $119=0,70 \mathrm{p}<=0,49$, Tabla 3C), pero al comparar entre las cuatro grupos si hubo diferencias en los valores promedio (F3, $119=19,64 \mathrm{p}<=0,0001$, Tabla 3C). Además, la triple interacción unidades médicas $\times$ cuatro grupos $\times$ la covariable CP1 muestra que el Grupo 1 (mujeres no jefas de familia) y el Grupo 3 (hombres jefes de familia) de las tres unidades médicas tuvieron un GBS promedio alto y el estado civil correspondió a estar casados oenunión libre. Sin embargo, destaca el registro en el Grupo 2 (hombres no jefes de familia) y el Grupo 4 (mujeres jefas de familia) que son solteros, fueron los grupos con menor GBS (ANCOVA F 2,119=8, $\mathrm{r} 2=0,11 \mathrm{p}<=0,0001$, Figure $2 \mathrm{C}$ ). 


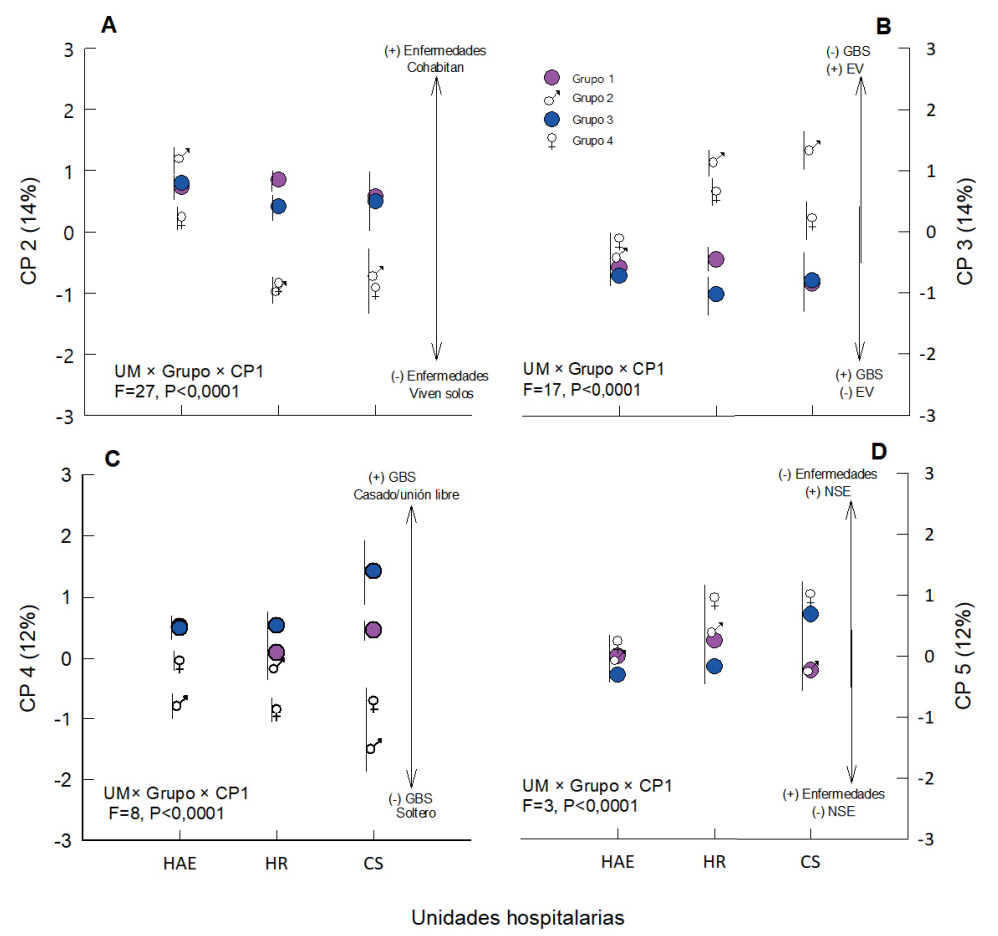

Figura $\mathbf{N}^{\circ}$ 2: Comparación de los valores promedio de los componentes principales $\mathrm{CP} 2$ a CP5 para la interacción entre Unidad médica $\times$ Cuatro grupos $\times$ covariable CP1. Las barras verticales indican un error estándar. Las abreviaturas son las siguientes: HAE $=$ Hospital de Alta Especialidad, $\mathrm{HR}=$ Hospital Regional, $\mathrm{CS}=$ Centro de Salud. Grupo $1=$ mujeres no jefas de familia, Grupo 2= hombres no jefes de familia, Grupos 3= hombres jefes de familia y Grupos 4= mujeres jefas de familia. Las flechas indican las características de los sujetos de acuerdo al Componente Principal (CP).

El CP5, producto de la combinación lineal entre la enfermedad y nivel socioeconómico, muestra que los valores promedio contrastan entre unidades médicas (F2, 119=4,83 $\mathrm{p}<=0,001$, Tabla 3D), los cuatro grupos también contrastaron (F3, 119=4,93 $\mathrm{p}<=0,001$, Tabla 3D) y la triple interacción mostró diferencias en las unidades médicas $\times$ cuatro grupos $\times$ la covariable $\mathrm{CP} 1(\mathrm{~F} 12,119=3,22 \mathrm{p}<=0,0001$, Tabla 3D). Esta última interacción delimita que la población estudiada en el HAE presentaban una mayor carga de enfermedad y un nivel socioeconómico bajo. El Grupo 4 (mujeres jefas de familia) en el HR y al CS tienen la menor carga de enfermedad y un nivel socioeconómico medio(ANCOVA F2,119=3, $\mathrm{r} 2=0,08 \mathrm{p}<=0,0001$, Figura 2D).

La caracterización que se obtuvo de los AM fue la conformaciónde cuatro grupos: hombres jefes de familia y mujeresno jefas de familia quienes registraron estilos de vida malos, un GBS promedio más alto, asociación de varias enfermedades, un nivel socioeconómico bajo y ello se asociaciónestar casado o en unión libre y cohabitar con otras personas en el hogar. En cambio, los otros dos grupos se integraron por hombres no jefes de familia y mujeres jefas de familia que presentaron mejores estilos de vida, un promedio de GBS menor, una sola enfermedad, nivel socioeconómico medio, pero suelen vivir solos. 
Los resultados indican que la combinación de variables integradas en índices multivariados delimitan cuatro grupos de adultos mayores y la condición que definió dicho agrupamiento fue el hecho de ser jefe de familia, además las mujeres que llegaron a los 60 años asumían un rol de jefas y proveedoras. Sin embargo, se delimitó un grupo de hombres que dependen de la familia. En este sentido, las mujeres en este estudio han adoptado el rol de jefas y proveedoras, puesto que en la vejez los roles se modifican debido a que, se retiran del trabajo, cambia la capacidad económica, y el deterioro de la salud origina la dependencia hacia la familia para su manutención y cuidado.

El índice multivariado del CP2 y su interacción con la unidad médica, ratifica mientras más enfermedades padezcan los $\mathrm{AM}$,estos son referidos a una unidad médica más especializada, donde la combinación de padecimientos complica su control. Lo anterior confirma la necesidad de crear redes integradas de salud, para mejorar la continuidad y el manejo integral de la atención de pacientes con enfermedad crónica con énfasis en los procesos de referencia y contra referencia, con la finalidad de ofrecer un mejor control al paciente AM que vive con una enfermedad crónica, y de esta forma contribuir a disminuir el GBS(16).

A partir de la caracterización de los AM en función de su vulnerabilidad, donde los hombres son jefes de familia y/o las mujeres no son jefas de familia, resulta fundamental, fomentar el diseño políticas públicas así como la implementación programas verticales orientados a promover estilos de vida saludables, y la continuidad de la atención, para mejorar la capacidad resolutiva, prevenir la presencia de complicaciones de las enfermedades que padecen los adultos mayores mediante la detección temprana y evitar la referencia a instituciones de mayor complejidad, lo cual les ocasiona GBS.

Es importante destacar, que los hombres y mujeres que tenían pareja y vivían con familiares (hijos, nueras o yernos, nietos), tuvieronestilos de vida malos, padecían varias enfermedades y registraron un GBS promedio más alto, lo cual es contradictorio puesto que se esperaría encontrarlo en los AM que vivían solos.El hecho de cohabitar con otros miembros de la familia,apoya a cubrir los gastos generados en la atención de este tipo de población(17). Sin embargo, lo que ocurre es que el rol que asumen los AM y sobre todo las mujeres, es de cuidadora familiar (nietos y/o pareja enferma), además de realizar el trabajo doméstico, que en ocasiones no es valorado, lo que conlleva a invertir poco tiempo en su autocuidado. Asimismo el maltrato de los adultos por parte de las personas con las que habitan está relacionado con la presencia de un mayor número de patologías(18). Aunque en este estudio no se evaluó si la población de estudio tenía este tipo de características, sería interesante profundizar en esta problemática.

El estudio demuestra quemalos estilos de vida del AM en conjunto con un ingreso bajo, está asociado a un GBS alto, ya que las desigualdades en salud desfavorecen a la población con menores ingresos. Al respecto se coincide con estudios que demuestran la influencia de los factores socioeconómicos y/o estilos de vida con diversas enfermedades en los AM.En ese sentido algunas evidencias demuestran la asociación entre el bajo nivel socioeconómico con la demencia (19);individuos inactivos que presentan problemas económicos asociados con migraña(20);nivel socioeconómico y estilos de vida desfavorables con obesidad (21); y estilos de vida poco saludables con un nivel socioeconómico bajo y síntomas de depresión(22). En cambio, cuando hay mayor educación, apoyo de redes sociales, además de tener una ocupación después de la mitad de la vida se asocia con una mejor trayectoria cognitiva (23).

La fortaleza de este estudio es que al analizar múltiples variables se aporta un conocimiento integral de lo sucedido en poblaciones vulnerables y poco visibles para los sistemas de salud, como es el caso de los AM en condiciones socioeconómicas desfavorables. 
Limitaciones del estudio: En este estudio se analizaron las variables mencionadas en una red de servicios de salud pública en diferentes niveles de atención. En este sentido, se identifican limitaciones relacionadas con la población incluida en el mismo,en virtud de que no caracteriza a las diferentes instituciones públicas prestadoras de servicios de salud en México y a sus respectivos usuarios. Por tal motivo sería interesante que en investigaciones posteriores se ampliara a otras instituciones incluyendo un abordaje cualitativo que analice también la voz de los usuarios. Dada la importancia que reviste el GBS sería pertinente el diseño de estudios longitudinales que monitoren esta variable mediante trazadores en salud.

\section{Conclusiónes}

En conclusión, se logró la caracterización de cuatro grupos de AM de los cuales dos se colocan en una situación de mayor vulnerabilidad de acuerdo a los determinantes sociales a los que estuvieron expuestos, entre ellos el propio sistema de salud. Lamentablemente, el aseguramiento al que tienen derecho los AM, para el caso que nos ocupa mediante el Seguro Popular, no los protege financieramente provocándoles un GBS (24).

DECLARACIÓN SOBRE CONFLICTOS DE INTERÉS:Los autores declaran que no existe conflicto de interés.

\section{Bibliografía}

1. Pastor, Yolanda, Balaguer, Isabel y Garcia-Merita M. Una revisión sobre las variables de estilos de vida saludables. Rev Psicol la salud. 1998;10(1):15-52.

2. Iwamoto T. Prevention of dementia on the basis of modification of lifestyle and management of lifestyle-related diseases: a review. Nihon Rinsho. 2014;72(4):6127. Disponible en: https://www.ncbi.nlm.nih.gov/pubmed/24796088

3. Mumu S, Saleh F, Ara F, Afnan F, Ali L. Non-adherence to life-style modification and its factors among type 2 diabetic patients. Indian J Public Health [Internet]. 2014;58(1):40. Disponible en: http://www.ijph.in/text.asp?2014/58/1/40/128165

4. Johari SM, Shahar S. Metabolic Syndrome: The Association of Obesity and Unhealthy Lifestyle among Malaysian Elderly People. Arch Gerontol Geriatr [Internet]. 2014;59(2):360-6. http://dx.doi.org/10.1016/j.archger.2014.04.003

5. Giltay EJ, Geleijnse JM, Zitman FG, Buijsse B, Kromhout D. Lifestyle and dietary correlates of dispositional optimism in men: The Zutphen Elderly Study. J Psychosom Res. 2007;63(5):483-90. DOI: 10.1016/j.jpsychores.2007.07.014

6. Pala V, Sieri S, Masala G, Palli D, Panico S, Vineis P, et al. Associations between dietary pattern and lifestyle, anthropometry and other health indicators in the elderly participants of the EPIC-Italy cohort. Nutr Metab Cardiovasc Dis. 2006;16(3):186201. DOI: 10.1016/j.numecd.2005.05.009 P

7. Consejo Nacional de Población (CONAPO). Proyecciones de la población 20102030 [Internet]. México; 2017. Disponible en: www.conapo.com.mx

8. Organización Panamericana de la Salud. Salud en las Américas: edición 2012. Panorama regional y perfiles de País [Internet]. Washington, DC; 2012. Disponible en: file://Users/patriciapavonleon/Downloads/9789275316917_spa.pdf

9. Gutiérrez JP, Rivera-Dommarco J, Shamah-Levy T, Villalpando-Hernández S, Franco A, Cuevas-Nasu L, Romero-Martínez M H-ÁM. Encuesta Nacional de Salud y Nutrición 2012. Resultados Nacionales [Internet]. Instituto Nacional de Salud Pública. Cuernavaca, México: Instituto Nacional de Salud Pública (MX), 2012.; 2012. p. 200. Disponible en: http://ensanut.insp.mx/informes/ ENSANUT2012ResultadosNacionales.pdf

10. Organization WH. No Title [Internet]. Out-of-pocket payments, user fees and catastrophic expenditure. 2018 [cited 2018 Aug 10]. Disponibe en: http://www. 
11. AguilaE DíazC, Manqing M,ArieF, Ashloy K. EnvejecerEn México : Condiciones de Vida y Salud [Internet]. AARP/Rand Corporation/Centro Fox, editor. México; 2011. 1-128 p. Disponible en: http://coespo.qroo.gob.mx/Descargas/doc/15 ENVEJECIMIENTO POBLACIONAL/ENVEJECER EN MÉXICO.pdf

12. Bronfman M., Guiscafre H., Castro V., Castro R. GG. Measuring unequality: a methodological approach, analysis of social and economic characteristics of the sample studied. Arch Invest Med [Internet]. 1988;19(4):351-60. Disponible en: http://www.definiciones-de.com/Definicion/de/nivel_socioeconomico.php

13. López-Carmona JM, Rodríguez-Moctezuma R, Munguía-Miranda C, HernándezSantiago JL, de la Torre EC. Validez y fiabilidad del instrumento «FANTASTIC» para medir el estilo de vida en pacientes mexicanos con hipertensión arterial. Atención Primaria [Internet]. 2000;26(8):542-9. Disponible en: http://www. sciencedirect.com/science/article/pii/S0212656700787191

14. Cuadras Carles M. Nuevos métodos de análisis multivariante [Internet]. CMC Editions, editor. CMC Editions. Barcelona, España; 2014. 305 p. Disponible en: http://www.ub.edu/stat/personal/cuadras/metodos.pdf

15. Richard L. Gorsuch. Factor analysis. Second Edi. Hillsdale NLEA, editor. New Jersey London; 1983.

16. Organización Panamericana de la Salud. Redes Integradas de Servicios de Salud. Conceptos, Opciones de Política y Hoja de Ruta para su Implementación en las Américas. Área de Sistemas y Servicios de Salud (HSS). Equipo de Sistemas de Salud y Protección Social (HSS/SP). Serie La Renovación de la Atención Primaria de Salud en las Américas. Washington, D. C.: OPS; 2008.

17. Knaul F.M., Wong R., Arreola-Ornelas H., Méndez O. et al. Household catastrophic health expenditures: A comparative analysis of twelve latin American and Caribbean countries. Salud Publica Mex. 2011;53(SUPPL. 2):85-95. Disponible en: https:// www.ncbi.nlm.nih.gov/pubmed/21877097

18. Ruelas-González M.G. SZV. Factores asociados con el auto-reporte de maltrato en adultos mayores de México. Rev Chil Salud Pública. 2009;13(2):90-9. Disponible en:https://revistasaludpublica.uchile.cl/index.php/RCSP/article/view/642

19. Yaffe K, Falvey C, Harris TB, Newman A, Satterfield S, Koster A, et al. Effect of socioeconomic disparities on incidence of dementia among biracial older adults: prospective study. Bmj [Internet]. 2013;347(dec19 5):f7051-f7051. Disponible en: http://www.bmj.com/cgi/doi/10.1136/bmj.f7051

20. Molarius A, Tegelberg A, Ohrvik J. Socio-economic factors, lifestyle, and headache disorders - A population-based study in Sweden. Headache. 2008;48(10):1426-37. DOI: $10.1111 /$ j.1526-4610.2008.01178.x

21. Han TS, Lee DM, Lean MEJ, Finn JD, O’Neill TW, Bartfai G, et al. Associations of obesity with socioeconomic and lifestyle factors in middle-aged and elderly men: European Male Aging Study (EMAS). Eur J Endocrinol. 2015;172(1):59-67.DOI: 10.1530/EJE-14-0739

22. Groffen DAI, Koster A, Bosma H, Van Den Akker M, Kempen GIJM, Van Eijk JTM, et al. Unhealthy lifestyles do not mediate the relationship between socioeconomic status and incident depressive symptoms: The health ABC study. Am J Geriatr Psychiatry. 2013;21(7):664-74. DOI: 10.1016/j.jagp.2013.01.004

23. Marioni RE, Proust-Lima C, Amieva H, Brayne C, Matthews FE, Dartigues JF, et al. Cognitive lifestyle jointly predicts longitudinal cognitive decline and mortality risk. Eur J Epidemiol. 2014;29(3):211-9. DOI: 10.1007/s10654-014-9881-8

24. Pavón-León P, Reyes-Morales H, Martínez AJ, Méndez-Maín SM, GogeascoecheaTrejo M delC, Blázquez-Morales MSL. Gasto de bolsillo en adultos mayores afiliados a un seguro público de salud en México. Gac Sanit [Internet]. 2017;31(4):286-91. http://dx.doi.org/10.1016/j.gaceta.2016.12.015. 